\title{
Displaced medial epicondyle fractures: retrospective study and review of the literature
}

\author{
Anna Maria Nucci', Filippo Maria Senes², \\ Annamaria Dell'Unto ${ }^{3}$, Silvio Boero ${ }^{4}$ \\ ${ }^{1}$ Traumatology and Orthopedics Unit, Azienda Ospedaliera Universitaria Pisana, \\ University of Pisa, Italy; ${ }^{2}$ Hand Surgery and Reconstructive Microsurgery Unit, IRCCS \\ Istituto "G. Gaslini", Genoa, Italy; ${ }^{3}$ Traumatology and Orthopedics Unit, Azienda \\ Ospedaliera Universitaria Policlinico Umberto I di Roma, Sapienza Università di Roma, \\ Rome, Italy; ${ }^{4}$ Department of Paediatric Orthopaedics, IRCCS Istituto "G. Gaslini", \\ Genoa, Italy
}

\section{SUMMARY}

Objective. To present the clinical and functional results of medial epicondylar fractures in 24 surgically-treated children with long-term follow-up.

Methods. We retrospectively reviewed 24 cases of displaced medial epicondylar fracture surgically treated between January 2013 and December 2016. Demographical characteristics of patients were recorded and analyzed along with radiographical images. Patients underwent clinical evaluation and were asked to answer to the Quick DASH test to assess long term functional outcome.

Received: July 25, 2020

Accepted: September 10, 2020

\section{Correspondence}

Anna Maria Nucci

Traumatology and Orthopedics Unit, Azienda Ospedaliera Universitaria Pisana, University of Pisa, via Paradisa 2, Pisa, Italy

E-mail: annamarianucci90@gmail.com

Conflict of interest

The Authors declare no conflict of interest

How to cite this article: Nucci AM, Senes FM, Dell'Unto A, et al. Displaced medial epicondyle fractures: retrospective study and review of the literature. Lo Scalpello Journal 2020;34:137-9. https://doi.org/10.36149/0390-5276-168

(C) Ortopedici Traumatologi Ospedalieri d'Italia (O.T.O.D.I.) 2020

\section{(c) (1) (8) $\Theta$}

This is an open access article distributed in accordance with the CC-BY-NC-ND (Creative Commons Attribution-NonCommercial-NoDerivatives 4.0 International) license. The article can be used by giving appropriate credit and mentioning the license, but only for non-commercial purposes and only in the original version. For further information: https://creativecommons.org/licenses/by-nc-nd/4.0/deed.en
Results. Mean follow-up was 4.8 years. Complete range of movement of the elbow was restored in all cases except for 4 cases of extension lag inferior to $30^{\circ}$ and 2 cases of slightly reduced pronation. Valgus deformity of the elbow inferior to $15^{\circ}$ was observed in 3 patients. Mean Quick DASH score was 15.1.

Conclusions. Even if the treatment guidelines of displaced medial epicondyle fractures are still debated, our study demonstrates satisfying functional results in the long term after surgical treatment, without major complications. In addition, surgery allows anatomic reduction of the fracture, preventing fibrous nonunion.

Key words: elbow, humeral fractures, children

\section{Introduction}

Medial epicondyle fractures are the third most common elbow lesion in children after supracondylar and lateral condyle fractures ${ }^{1}$. Up to $60 \%$ of cases are associated with elbow dislocation ${ }^{2}$. The medial epicondyle is an extra-articular apophysis which is the site of insertion of the pronator-flexor muscular mass and the medial collateral ligament complex of the elbow. Its nucleus of ossification appears at about 5 years in females and 7 years in males and is the last to fuse between 15 and 18 years. Two main traumatic mechanisms have been described. The first implies a direct blow on the medial aspect of the elbow resulting in a fragmented medial epicondyle. However, the most widely accepted is an avulsion mechanism produced by a valgus stress on the extended elbow ${ }^{3}$. Nonoperative treatment represents the first choice in case of nondisplaced or minimally displaced fractures. Nevertheless, the degree of displacement beyond which surgical treatment is indicated has not yet been defined. On the other hand, surgical intervention represents an absolute indi- 
cation in case of incarcerated epicondylar fragment and open fractures. Ulnar nerve entrapment, gross elbow instability, and fractures in high-demanding patients are considered relative indications ${ }^{2}$.

The purpose of this paper is to present the results of open reduction and fixation of displaced medial epicondylar fractures in 24 patients associated with a literature review on the subject.

\section{Materials and methods}

This retrospective study reviewed 42 patients who were diagnosed with a medial epicondylar fracture between January 2013 and December 2016 in the Orthopedic and Traumatology Unit of the Scientific Institute G. Gaslini in Genoa. Sixteen fractures with a fragment displacement inferior to $5 \mathrm{~mm}$ were considered nondisplaced and excluded. The other 26 patients with a displacement superior to $5 \mathrm{~mm}$ were categorized as displaced medial epicondylar fracture. Twenty-four of these were included in the study, while two patients were lost to follow-up. Clinical data and radiographical images of each patient were retrieved. In particular, patient age, side and traumatic mechanism of the fracture, time and type of surgical intervention, postoperative complications were registered and analyzed. Pre- and postoperative radiographical images were evaluated in terms of degree of displacement and association with other bone lesions. All patients underwent clinical evaluation. Elbow stability, articular range of movement, and upper limb axis were assessed and compared to the healthy side. Quick Dash test was administered in order to estimate functional outcome. The score ranges from 11 (least disability) to 55 points (most disability). Written informed consent was obtained from adult patients or, in case of minor patients, from the parents or the legal guardians.

\section{Results}

Twenty-four patients were included in the study. There were 15 females and 9 males with a mean age of 11.6 years (range 9-13) at the time of the fracture. Mean follow-up was 4.8 years (range 4-6.1). As for the side, 11 patients injured their left elbow and 13 patients the right elbow. The dominant upper limb was involved in 9 cases. The trauma was sport related in 15 cases, while the other patients reported the fracture after an accidental fall. In one case there was a concomitant nondisplaced radial head fracture and in one case an elbow dislocation. The mean degree of displacement measured on X-rays was $6.8 \mathrm{~mm}$ (range 5.3-7.4). Mean time from admission to surgery was 1.5 days (range 0-4). A closed reduction was obtained in 6 cases, while in 18 patients a medial approach to the elbow was performed. Osteosynthesis was obtained by means of Kirschner wires or screws. Kirschner wires were removed at one month after surgery. All fractures were radiographically and clinically healed in 3 months. No major postoperative complication occurred except for one case of tingling in the areas innervated by the ulnar nerve, which spontaneously remitted after 2 years. In terms of clinical results, normal elbow range of movement was restored in all cases except for 4 cases of extension lag inferior to $30^{\circ}$ and 2 cases of slightly reduced pronation (reduction of $5^{\circ}$ ). In 3 cases a valgus deformity of the elbow was observed compared to the uninjured side $\left(8^{\circ}, 10^{\circ}\right.$ and $\left.10^{\circ}\right)$. No cases of elbow instability were found. Mean Quick DASH score was 15.1 (range 11-31). Full activity of the elbow was restored in all cases.

\section{Discussion}

Treatment of displaced medial epicondyle fracture in children is still debated. Although there is a large amount of literature on the subject, the amount of displacement that marks the limit between surgical and conservative treatment has not yet been defined. Some authors consider surgical intervention for displacements just superior to $2 \mathrm{~mm}^{4}$, while others prefer conservative treatment until $10 \mathrm{~mm}$ of displacement ${ }^{5}$. Moreover, the common practice of measuring displacement on elbow standard radiographs presents low intraobserver and interobserver agreement, as demonstrated by Pappas et al. ${ }^{6}$. As a consequence, the use of fragment displacement as a criterion for choosing treatment strategy appears unreliable. One of the main concerns after a medial epicondylar fracture is the development of elbow instability, as this is the site of insertion of the medial collateral ligament of the elbow. It has been demonstrated that nonoperative treatment leads to fibrous non-union of the epicondylar fragment in about $90 \%$ of cases. The real effect of medial epicondyle nonunion on elbow stability is also debated. Some studies demonstrated that, despite the high prevalence of nonunion after conservative treatment, patients present good functional and clinical results without pain or instability ${ }^{7}$. On the other hand, some authors have reported cases of painful nonunion and functional impairment after cast immobilization ${ }^{8}$. According to Shukla et al., medial epicondylar nonunion could be cause of valgus instability, especially in high demanding patients like gymnasts and throwing athletes ${ }^{9,10}$. Advocates of operative treatment sustain that surgical treatment of displaced fractures has the advantage to allow an anatomic reduction, guaranteeing a high union rate and preventing elbow instability ${ }^{11}$. Obviously, operative treatment implies all the risks of surgical intervention.

In our study, operative treatment was adopted in 24 cases of medial epicondylar fracture with displacement superior to $5 \mathrm{~mm}$, after carefully explaining to parents the advantages and disadvantages of both nonoperative and operative treatment. Our results show satisfying long term clinical and functional outcomes, without major complications. Slight valgus deformity (inferior to $10^{\circ}$ ) was found in 3 patients, but in the absence of instability or pain. All patients had a complete radiograph- 
ical fracture consolidation in 3 months and, after a mean follow-up of 4.8 years, demonstrated a full functional recovery, as demonstrated by the mean Quick DASH score ${ }^{12}$.

\section{Conclusions}

The treatment for displaced medial epicondyle is still controversial. In our study, surgical treatment was an effective and safe therapeutic option even in the long term, reducing the risk of developing fibrous nonunion and elbow instability.

\section{Acknowledgements}

No funds were received in support of this study.

\section{References}

1 Mehlman CT, Howard AW. Medial epicondyle fractures in children: clinical decision making in the face of uncertainty. J Pediatr Orthop 2012;32:135-42. https://doi.org/10.1097/ BPO.0b013e31824bdb78

2 Gottschalk HP, Eisner E, Hosalkar HS. Medial epicondyle fractures in the pediatric population. J Am Acad Orthop Surg 2012;20:223-32. https://doi.org/10.5435/ JAAOS-20-04-223

3 Firth AM, Marson BA, Hunter JB. Paediatric medial humeral epicondyle fracture management: 2019 approach. Curr Opin Pediatr 2019;31:86-91. https://doi.org/10.1097/MOP.0000000000000707

4 Hines RF, Herndon WA, Evans JP. Operative treatment of medial epicondyle fractures in children. Clin Orthop Relat Res 1987;223:1704. https://doi.org/10.1097/00003086-198710000-00019
5 Knapik DM, Fausett CL, Gilmore A, et al. Outcomes of nonoperative pediatric medial humeral epicondyle fractures with and without associated elbow dislocation. J Pediatr Orthop 2017;37:224-8. https://doi.org/10.1097/BPO.0000000000000890

6 Pappas N, Lawrence JT, Donegan D, et al. Intraobserver and interobserver agreement in the measurement of displaced humeral medial epicondyle fractures in children. J Bone Jt Surg - Ser A 2010;92:322-7. https://doi.org/10.2106/JBJS.I.00493

7 Farsetti P, Potenza V, Caterini R, et al. Long-term results of treatment of fractures of the medial humeral epicondyle in children. J Bone Jt Surg 2001;83-A:1299-305. https://doi. org/10.2106/00004623-200109000-00001

8 Smith J, McFeely E, Bae D, et al. Operative fixation of medial humeral epicondyle fracture nonunion in children. J Pediatr Orthop 2010;30:644-8. https://doi.org/10.1097/BPO.0b013e3181ed4381.

9 Shukla SK, Cohen MS. Symptomatic medial epicondyle nonunion: treatment by open reduction and fixation with a tension band construct. J Shoulder Elb Surg 2011;20:455-60. https://doi. org/10.1016/j.jse.2010.12.017

10 Chorney M, Sylvestre D, Seeley M. Medial epicondyle nonunions in children. JAAOS Glob Res Rev 2019;3:e046. https://doi. org/10.5435/jaaosglobal-d-18-00046

11 Louahem DM, Bourelle S, Buscayret F, et al. Displaced medial epicondyle fractures of the humerus: surgical treatment and results. A report of 139 cases. Arch Orthop Trauma Surg 2010;130:64955. https://doi.org/10.1007/s00402-009-1009-3

12 Canavese F, Marengo L, Tiris A, et al. Radiological, clinical and functional evaluation using the Quick Disabilities of the Arm, Shoulder and Hand questionnaire of children with medial epicondyle fractures treated surgically. Int Orthop 2017;41:1447-52. https://doi.org/10.1007/s00264-017-3442-1 